\title{
Prática de ioga como terapia complementar ou alternativa em crianças e adolescentes com perturbação de hiperatividade e défice de atenção: uma revisão baseada na evidência
}

Inês Pintalhão ${ }^{1}$, Joana Penetra², Joel Batista ${ }^{3}$

\section{RESUMO}

Objetivo: Rever a evidência disponível acerca do efeito da prática de ioga na diminuição dos sintomas de perturbação de hiperatividade e défice de atenção (PHDA) em crianças e adolescentes.

Fontes de dados: MEDLINE/PubMed e bases de dados de medicina baseada na evidência (National Guideline Clearinghouse, Canadian Medical Association Practice Guidelines InfoBase, Guidelines Finder da National Electronic Library for Health - NHS britânico, DARE, Bandolier e The Cochrane Library).

Métodos de revisão: Pesquisa de artigos (normas de orientação clínica, meta-análises, revisões sistemáticas e estudos originais) publicados entre janeiro de 2000 e janeiro de 2017 nas línguas portuguesa e inglesa, utilizando os termos MeSH attention deficit hyperactivity disorder, yoga, child e adolescent. O nível de evidência e a força de recomendação foram atribuídos de acordo com os critérios da escala Strength of Recommendation Taxonomy (SORT), da American Family Physician.

Resultados: Foram encontrados 48 artigos no total, dos quais se selecionaram cinco de acordo com os critérios de inclusão: uma revisão sistemática e quatro artigos originais, entre os quais três ensaios clínicos controlados e aleatorizados e um ensaio quase-experimental. Apesar de alguns estudos apresentarem resultados positivos, não há evidência suficiente que demonstre efeito benéfico da prática de ioga na diminuição dos sintomas de PHDA (nível de evidência 2).

Conclusão: A evidência disponível não permite suportar com robustez a recomendação da prática de ioga em crianças e adolescentes com PHDA, como terapia alternativa ou complementar às estratégias já implementadas (força de recomendação $B$ ). Os estudos que atribuem efeito benéfico à modalidade levantam preocupações quanto à metodologia, limitando a qualidade da evidência. Sugere-se a realização de mais estudos prospetivos e de larga escala, com amostras homogéneas e follow-up adequado, que a longo prazo permitam validar a evidência encontrada e auxiliar na formulação de recomendações.

Palavras-chave: Perturbação de hiperatividade e défice de atenção; loga; Criança; Adolescente.

\section{INTRODUÇÃO}

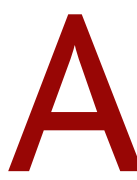
perturbação de hiperatividade e défice de atenção (PHDA) é uma das perturbações neurocomportamentais mais frequentes na criança e no adolescente ${ }^{1-3}$ que pode persistir ao longo da vida, acarretando várias comorbilidades. De acordo com o MANUAL DE DiAgNóstico E Estatístico
DE DoenÇAs MENTAIS, versão 5 (DSM-5), ${ }^{4}$ a PHDA é caracterizada por um padrão persistente de desatenção e/ou hiperatividade/impulsividade, que interfere no

1. USF Garcia de Orta, ACeS Porto Ocidental

2. USF Topázio, ACeS Baixo Mondego

3. UCSP Cinfães, ACeS Tâmega I, Baixo Tâmega 
normal funcionamento e desenvolvimento, tendo impacto negativo nas atividades sociais, académicas e profissionais. Na ClassifiCAÇÃo INTERNACIONAL DE DOENÇAS, versão 10 (CID-10), a nomenclatura diverge sendo denominada perturbação hipercinética, mas os critérios de diagnóstico são semelhantes. ${ }^{5}$

A prevalência da PHDA varia de acordo com a população estudada e com os critérios de diagnóstico, embora não existam dados precisos para as crianças portuguesas. O DSM-5 aponta uma prevalência de cerca de $5 \%$ em crianças e adolescentes, sendo mais comum no género masculino com uma proporção de 2:1. ${ }^{4}$ Estima-se que a patologia prevaleça no adulto em cerca de $60 \%$ dos casos. ${ }^{1,6}$ Apesar de a etiologia da PHDA permanecer desconhecida, existe uma componente multifatorial que envolve fatores genéticos, psicológicos, sociais e ambientais. $^{2}$ As manifestações clínicas geralmente modificam com a idade escolar, sendo mais evidentes na idade pré-escolar os sintomas de hiperatividade e impulsividade, que se atenuam na idade escolar e adolescência, dando lugar a sintomas relacionados com o défice de atenção.? Para além destes sintomas nucleares e/ou primários existem ainda défices em múltiplas áreas relacionadas com funções cerebrais executivas (planeamento, organização e memória de trabalho), atrasos de desenvolvimento e dificuldades na regulação emocional e comportamental. ${ }^{8}$ Estão disponíveis inúmeras escalas e questionários, validados para diferentes países, que permitem quantificar comportamentos e avaliar corretamente o diagnóstico e monitorização da evolução da PHDA, tanto para as crianças como para pais e educadores.

O tratamento da PHDA inclui uma abordagem educacional, psicossocial e farmacológica, que pode ser complementada por técnicas psicoterapêuticas cognitivo-comportamentais, programas de treino parental e treino de competências sociais. A farmacoterapia é em muitos casos o tratamento de eleição para a PHDA, com indicação do metilfenidato e da atomoxetina como fármacos de primeira linha. Geralmente a toma do fármaco é realizada durante o período escolar, em que é requerida maior atenção. No entanto, em crianças com alterações do comportamento mais sérias pode ser realizada durante o período de férias ou fins-de-semana. Numa sociedade em que a pressão para o diagnóstico de hiperatividade por parte dos pais e professores tem vindo a aumentar, ${ }^{9-10}$ torna-se fundamental a assertivi- dade no diagnóstico correto, evitando os riscos inerentes ao excesso de medicação de forma desnecessária.

Assim, na tentativa de encontrar estratégias complementares à terapia farmacológica, a prática de ioga tem ganho uma posição de destaque, tanto no domínio físico como psicológico. Os longos períodos de concentração exigidos pelo ioga, assim como o treino de relaxamento e de técnicas respiratórias são apontados como os principais motivos que poderão estar na base deste fundamento. ${ }^{11,13-14}$ Outros estudos indicam ainda que o ioga capacita para uma maior resistência a eventos stressantes, ${ }^{11,14}$ diminuindo a ativação do sistema nervoso simpático com a consequente ativação do parassimpático, provocando uma sensação de autocontrolo emocional., ${ }^{6,12-13}$

No entanto, pouco se conhece ainda acerca da eficácia desta estratégia na diminuição dos sintomas da PHDA. ${ }^{2} \mathrm{O}$ objetivo deste trabalho é rever a evidência disponível sobre o efeito da prática de um treino de ioga na diminuição dos sintomas da PHDA, como tratamento alternativo ou complementar às estratégias terapêuticas já conhecidas.

\section{MÉTODOS}

Pesquisa bibliográfica de artigos escritos em língua portuguesa ou inglesa (normas de orientação clínica, meta-análises, revisões sistemáticas e estudos originais) nas bases de dados MEDLINE/PubMed, National Guideline Clearinghouse, Guidelines Finder, Canadian Medical Association Practice Guideline Infobase, The Cochrane Library, Bandolier, bem como referências bibliográficas dos artigos selecionados. Foram utilizados os termos MeSH attention deficit hyperactivity disorder, yoga, child e adolescent-tendo sido pesquisados artigos publicados de 1 de janeiro de 2000 a 31 de janeiro de 2017.

Foram incluídos estudos segundo os critérios de inclusão definidos pelo modelo PICO:

1) População: crianças e adolescentes entre os cinco e os 18 anos de idade com diagnóstico de PHDA pelo DSM, versão 4 ou 5 (DSM 4/5) e/ou CID, versão 10 (CID-10), com ou sem tratamento farmacológico habitual;

2) Intervenção: treino de ioga;

3) Comparação: grupo placebo, ausência de intervenção; 


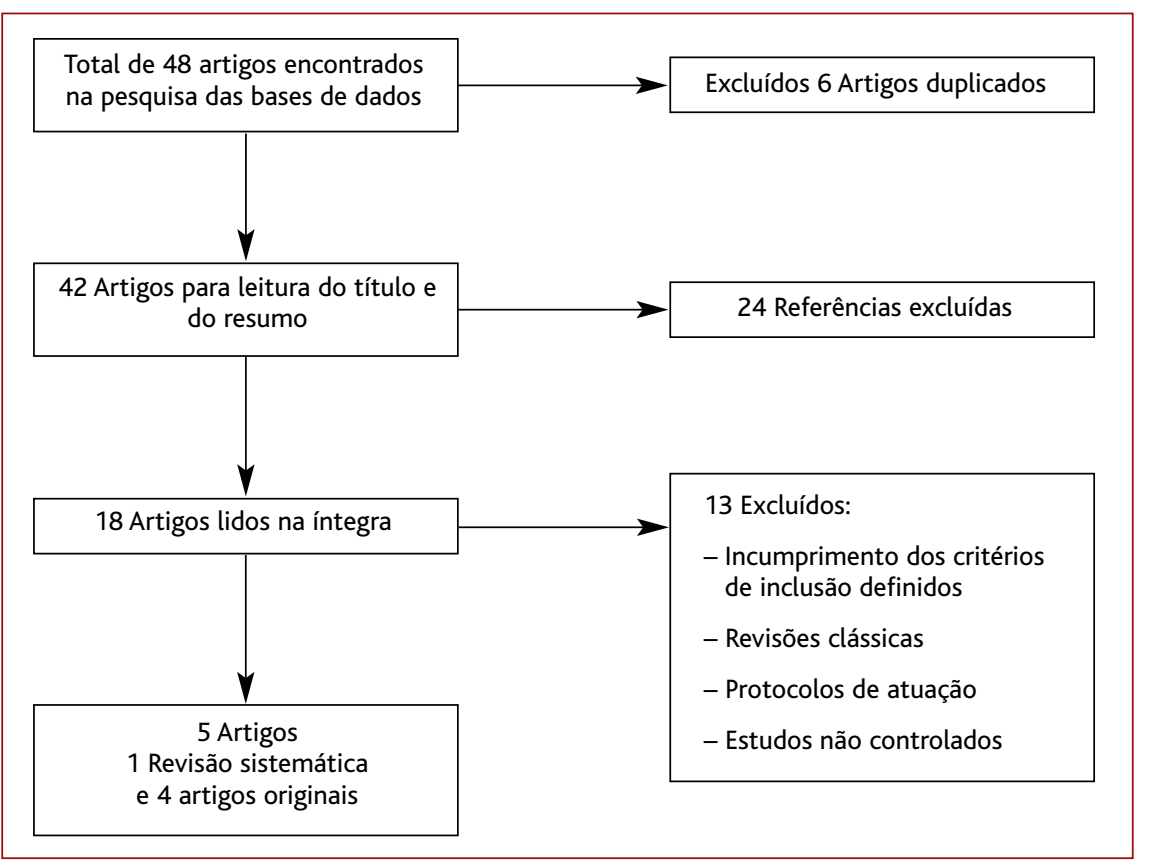

Figura 1. Esquema de seleção de artigos.

4) Outcome: diminuição dos sintomas primários e secundários da PHDA.

Foram excluídas crianças com outras comorbilidades psiquiátricas, doenças crónicas e/ou doenças fisicamente incapacitantes, assim como prática adicional de terapia comportamental ou outras técnicas de meditação.

Os artigos selecionados para leitura integral foram lidos por todos os autores para determinar a sua inclusão e a avaliação final da qualidade e nível de evidência foi discutida e decidida unanimemente.

Para atribuição dos níveis de evidência (NE) e força de recomendação (FR) foi utilizada a escala Strength of Recommendation Taxonomy (SORT), da American Family Physician. ${ }^{15}$

\section{RESULTADOS}

A pesquisa bibliográfica incluiu um total de 48 artigos, dos quais foram selecionados cinco: uma revisão sistemática (RS) e quatro artigos originais, dos quais três ensaios clínicos aleatorizados e controlados (ECAC) e um ensaio quase-experimental. O processo de seleção dos estudos está apresentado no fluxograma da Figura 1 e os resultados dos mesmos estão sucintamente descritos nos Quadros I e II.

\section{Revisões sistemáticas}

A revisão sistemática realizada por Balasubramaniam e colaboradores (de 2013) ${ }^{12}$ incluiu dois ECAC que avaliaram o efeito da prática de ioga em crianças com PHDA, envolvendo no total 35 crianças das quais 22 estavam sob medicação. No estudo alemão de Haffner e colaboradores (de 2006) ${ }^{3}$ foi comparado um grupo de crianças submetidas a um treino estruturado de ioga com um grupo que realizou exercício físico convencional e inespecífico durante 34 semanas. Os resultados foram avaliados para duas escalas alemãs, FBB-HKS Scale e Dortmund Attention Test. O outro ECAC, não duplamente cego, da autoria de Jensen e Kenny (de 2004) ${ }^{16}$ continha um grupo que foi submetido a um programa de ioga pré-definido e outro que envolvia atividades de cooperação (como treino de comunicação, escuta e partilha) durante 20 semanas. Os resultados foram avaliados segundo as respostas ao Questionário de Conners para Paise Professores. Ambos os estudos atribuíram mérito à prática de ioga como estratégia terapêutica complementar para crianças com PHDA, verificando-se melhoria significativa no período pós-intervenção para cada um dos parâmetros estudados para avaliar o outcome. Nenhum dos estudos revelou efeitos adversos ou prejudiciais com a prática de ioga. Tratam-se de ensaios não-duplamente cegos, sem processo de aleatorização bem definido, incluindo amostras pequenas e heterogéneas. Nenhum apresentou um programa de follow-up adequado. Atribuiu-se um nível de evidência 2 pela qualidade da metodologia efetuada pelos autores.

\section{Ensaios clínicos}

O mais recente estudo duplamente cego e quase experimental, da autoria de Chou e Huang (de 2017), ${ }^{6}$ foi realizado em 49 crianças, entre os oito e os 12 anos, com o objetivo de investigar o efeito da prática de um programa de ioga regular e estruturado nas funções executivas de crianças com PHDA. Do total, 22 crianças estavam medicadas. Durante oito semanas, um grupo foi 


\begin{tabular}{|c|c|c|c|c|}
\hline Referência & Estudos População & Intervenção & Resultados & NE \\
\hline $\begin{array}{l}\text { Balasubramaniam, } \\
\text { et al. } \\
(2013)^{12}\end{array}$ & $\begin{array}{l}2 \text { ECAC, não duplo-cegos } \\
n=35 \\
\text { Crianças diagnosticadas com } \\
\text { PHDA }\end{array}$ & $\begin{array}{l}\text { 1. loga vs exercício físico } \\
\text { convencional e inespecífico; } \\
\text { 2. loga vs atividades de } \\
\text { cooperação (treino de } \\
\text { comunicação, escuta e partilha) }\end{array}$ & $\begin{array}{l}\text { 1. O treino de ioga demonstrou } \\
\text { ser superior aos exercícios } \\
\text { motores convencionais com } \\
\text { efeito intervenção no intervalo } \\
\text { médio a alto }(0,60-0,97) \\
\text { 2. Grupo praticante de ioga } \\
\text { apresentou melhoria } \\
\text { significativa em cinco das } \\
\text { subescalas do CPRS }\end{array}$ & 2 \\
\hline
\end{tabular}

Legenda: $\mathrm{ECAC}=$ Ensaio clínico aleatorizado e controlado; PHDA = Perturbação de hiperatividade e défice de atenção; NE = Nível de evidência; CPRS = Conners' Parent Rating Scale.

submetido a um treino de ioga após a escola (40 min por sessão, duas vezes por semana) que incluía exercícios de flexibilidade, relaxamento e concentração. O grupo controlo não sofreu intervenção, sendo as crianças submetidas às suas atividades habituais depois do período escolar. Antes e depois do programa de intervenção os resultados foram estudados para dois testes: Visual Pursuit Test (VPT) para avaliação da perceção visual e atenção seletiva e Determination Test (DT), elaborado para testar o desempenho nas funções cognitivas. As crianças sob tratamento farmacológico foram instruídas para suspender a medicação $24 \mathrm{~h}$ antes da realização dos testes, antes e depois do programa. Para ambas as escalas, o grupo praticante de ioga apresentou um tempo de reação mais rápido $(p<0,001$ no VPT e DT) e taxa de precisão superior ( $p=0,045$ no VPT e $p<0,001$ no DT) no final da intervenção. Os resultados sugerem que a prática de exercício físico, complementada com treino de concentração, controlo corporal e desempenho motor, traduz o efeito benéfico da modalidade do ioga nas funções discriminativas e executivas das crianças com PHDA. Apesar de o estudo incluir uma amostra controlada e homogénea entre os dois grupos de comparação, o facto de ser não-randomizado limita a validade dos resultados pelo menor controlo das condições de exposição. Atribuiu-se, por isso, um nível de evidência 2.

O ECAC de Abadi e colaboradores (de 2008) ${ }^{13}$ apresentou um estudo em crianças iranianas com o objetivo de determinar o papel que a prática de ioga pode ter no tratamento dos sintomas de PHDA durante um programa de oito semanas que envolveu 40 crianças com idade média próxima dos 10 anos. Foi incluído um grupo experimental submetido a duas sessões de $45 \mathrm{~min}$ de ioga por semana durante o tempo estabelecido. $\mathrm{O}$ treino englobava exercícios de aquecimento muscular e técnicas respiratórias, treino postural específico e alongamentos e técnicas de relaxamento. O grupo controlo não sofreu intervenção de qualquer tipo. A ambos foi aplicada uma subescala de um questionário que mede os sintomas de PHDA (Child Symptoms Inventory) no período pré e pós intervenção. Os resultados concluíram que o grupo praticante de ioga diminuiu significativamente a pontuação do score do questionário $(\mathrm{F}=47,15(1,32)$; $p<0,001)$ em comparação com o grupo sem intervenção, nomeadamente nas questões que se destinavam a avaliar simultaneamente sintomas de atenção e impulsividade $(p<0,005)$. Não foi explicitado o número de crianças submetidas, ou não, a tratamento farmacológico. Apesar de o estudo utilizar uma amostra homogénea e de tamanho adequado, o procedimento de aleatorização e o método de ocultação não foram descritos. O método de seleção da amostra por conglomerados (cluster sampling) está sujeito a maior erro de amostragem, em termos de controlo de amplitude da amostra. Foi, por isso, atribuído um nível de evidência 2.

O ECAC da autoria de Haffner e colaboradores (de 2006), ${ }^{3}$ e já referido anteriormente na RS, pretendeu comparar a eficácia entre a prática de ioga e a prática de 


\begin{tabular}{|c|c|c|c|c|c|c|c|}
\hline Referência & $\begin{array}{l}\text { Tipo de } \\
\text { estudo }\end{array}$ & População & Intervenção & Duração & Outcome & Resultados & NE \\
\hline $\begin{array}{l}\text { Chou and } \\
\text { Huang } \\
(2017)^{6}\end{array}$ & $\begin{array}{l}\text { Quase- } \\
\text {-experi- } \\
\text { mental }\end{array}$ & $\begin{array}{l}\text { Crianças (entre } \\
\text { 8-12 anos) } \\
\text { PHDA (DSM-V) } \\
\text { - } n=49 \\
\text { (grupo controlo } \\
n=25 \text {; grupo } \\
\text { experimental } \\
n=24 \text { ) } \\
\text { - Idade média: } \\
10,5 \text { anos } \\
\text { - Género (M:F) } \\
=38: 11 \\
\text { Sob medicação: } \\
n=22\end{array}$ & $\begin{array}{l}\text { Prática de ioga } \\
\text { em grupo após a } \\
\text { escola ( } 40 \text { min } \\
\text { por sessão, } \\
2 x / \text { semana) } \\
\text { vs } \\
\text { Ausência de } \\
\text { intervenção } \\
\text { (atividades } \\
\text { habituais após } \\
\text { a escola) }\end{array}$ & 8 Semanas & $\begin{array}{l}\text { Visual Pursuit } \\
\text { Test (VPT) of } \\
\text { the Vienna Test } \\
\text { System } \\
\text { Determination } \\
\text { Test (DT) }\end{array}$ & $\begin{array}{l}\text { Para ambos os testes, no final } \\
\text { da intervenção, tempo de } \\
\text { reação mais rápido }(p<0,001) \\
\text { e taxa de precisão superior } \\
\text { ( } p=0,045 \text { no VPT; } p<0,001 \text { no } \\
\text { DT) no grupo praticante de } \\
\text { ioga. }\end{array}$ & 2 \\
\hline $\begin{array}{l}\text { Abadi, } \\
\text { et al. } \\
(2008)^{13}\end{array}$ & ECAC & $\begin{array}{l}\text { Crianças (entre } \\
9-12 \text { anos) com } \\
\text { PHDA (DSM-IV) } \\
\cdot n=40 \\
\text { (grupo controlo } \\
n=20 \text {; grupo } \\
\text { experimental } \\
n=20 \text { ) } \\
\text { - Idade média: } \\
\text { 10,1 anos } \\
\text { - Género: sem } \\
\text { referência }\end{array}$ & $\begin{array}{l}2 \text { Sessões de } \\
\text { ioga ( } 45 \mathrm{~min}) \\
\text { por semana } \\
\text { vs } \\
\text { Ausência de } \\
\text { intervenção }\end{array}$ & 8 Semanas & $\begin{array}{l}\text { Subescala do } \\
\text { Child Symptoms } \\
\text { Inventory } \\
\text { (CSI-4) }\end{array}$ & $\begin{array}{l}\text { O grupo praticante de ioga } \\
\text { diminuiu significativamente a } \\
\text { pontuação do score do } \\
\text { questionário ( } F=47,15[1,32] \\
p<0,001) \text {, em particular nas } \\
\text { questões que avaliavam } \\
\text { simultaneamente sintomas de } \\
\text { atenção e impulsividade } \\
(p<0,005) \text {. }\end{array}$ & 2 \\
\hline
\end{tabular}

exercício físico convencional e inespecífico na redução dos sintomas primários de PHDA num estudo cruzado durante um período de 34 semanas. O estudo incluiu dois grupos, um com 10 crianças que iniciaram o programa por treino de exercícios convencionais (corrida, «escondidas», jogos com bola, jogos sociais), seguido de treino de ioga, e outro com nove crianças que iniciaram o programa com a ordem inversa. Entre cada esquema de treino de oito semanas (duas vezes por semana), as crianças tinham seis semanas de descanso. Os resulta- dos foram estudados no pré e pós-teste para duas escalas alemãs que avaliam o desempenho em tarefas de atenção e os sintomas PHDA na visão dos pais e professores - FBB-HKS Scale que avalia sintomas de PHDA e Dortmund Attention Test (DAT) que testa a exatidão da observação e impulsividade cognitiva durante tarefas de perceção visual. Em ambas as escalas os resultados foram superiores após a prática de ioga do que após o exercício físico convencional inespecífico (FBB-HKS $p=0,007$, efeito intervenção 0,77 ; DAT $p<0,001$, efeito intervenção 


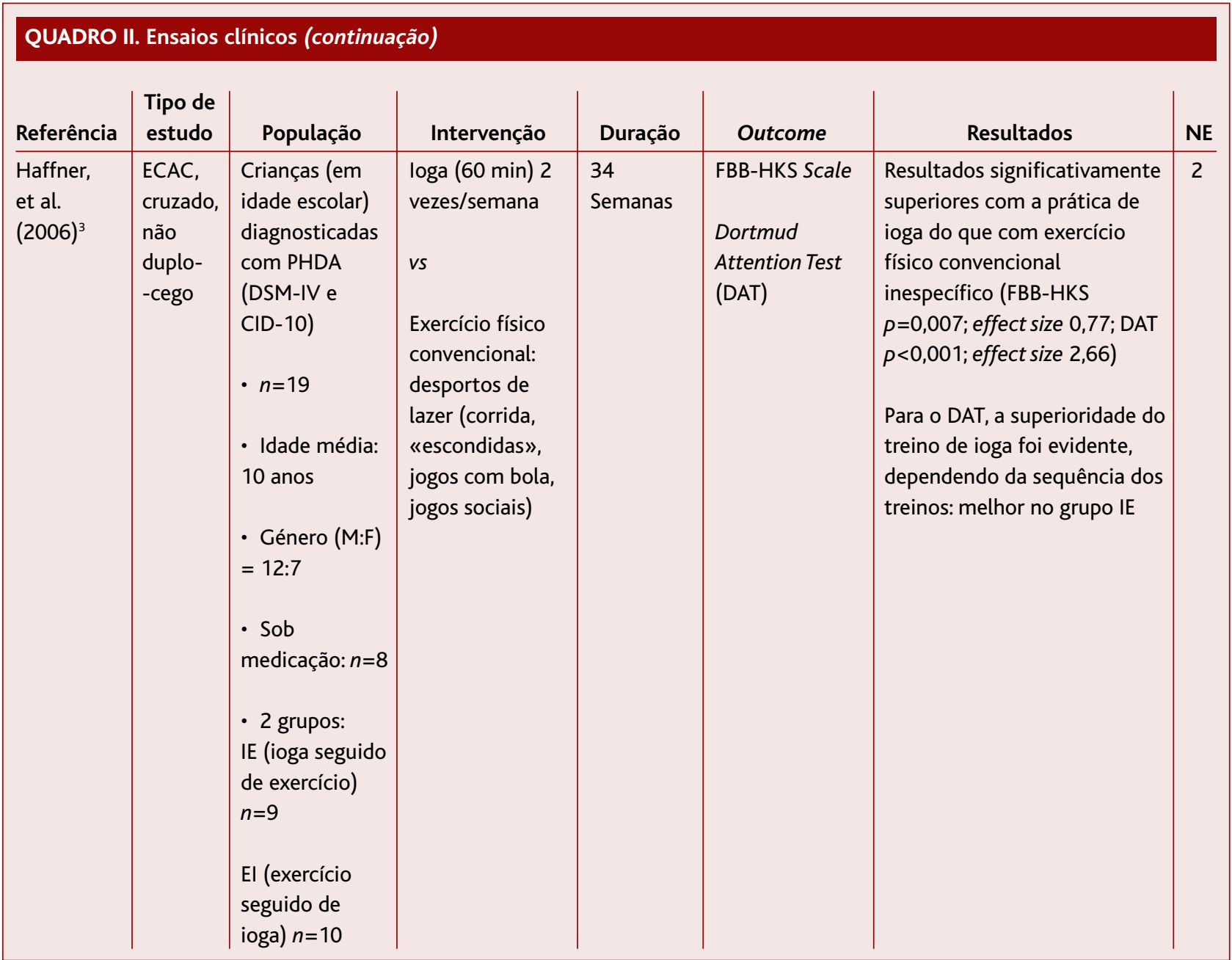

2,66). Para o DAT, a superioridade do treino de ioga foi evidente dependendo da sequência dos treinos: no grupo que iniciou o programa pelo treino de ioga verificou-se uma redução significativa dos sintomas para um nível não-patológico. As principais limitações do estudo consistem no facto de conter uma amostra pequena, sem dupla ocultação, não desenhar um programa de follow-up e de possuir um tempo curto entre as duas intervenções a fim de esperar a cessação do efeito residual (efeito carry-over). Assim, atribuiu-se um nível de evidência 2.

Jensen e Kenny (em 2004), ${ }^{16}$ também no ECAC já referido na RS, avaliaram os efeitos do ioga como tratamento complementar em crianças com PHDA medicadas e estáveis do ponto de vista clínico. Ao longo de 20 semanas foi comparado um grupo submetido a 20 ses- sões de ioga (uma hora por semana) com um grupo controlo que realizou exercícios e atividades de cooperação (atividades de comunicação, escuta e partilha), num total de 16 rapazes entre os oito e 13 anos. Os resultados pré e pós--intervenção foram avaliados para os Questionários de Conners para Pais e Professores, com a intenção de avaliar adicionalmente o comportamento dos filhos sem efeito de medicação (férias ou fins-de-semana). O grupo praticante de ioga mostrou, no final da intervenção, melhoria significativa em cinco das subescalas analisadas do Questionário de Conners para Pais, nomeadamente nas questões relativas à labilidade emocional ( $p=0,001$, Cohen's $d 0,79)$, oposição ( $p=0,003, C o$ hen's $d 0,77)$, impulsividade ( $p=0,008$, Cohen's $d 0,73)$, «índex global» $(p=0,019$, Cohen's $d 0,29)$ e «índex total» ( $p=0,001$, Cohen's $d 0,73$ ) em comparação com o grupo 


\section{QUADRO II. Ensaios clínicos (continuação)}

\begin{tabular}{|c|c|c|c|c|c|c|c|}
\hline Referência & $\begin{array}{l}\text { Tipo de } \\
\text { estudo }\end{array}$ & População & Intervenção & Duração & Outcome & Resultados & NE \\
\hline $\begin{array}{l}\text { Jensen and } \\
\text { Kenny } \\
(2004)^{16}\end{array}$ & $\begin{array}{l}\text { ECAC, } \\
\text { cruzado, } \\
\text { não } \\
\text { duplo- } \\
\text {-cego }\end{array}$ & $\begin{array}{l}\text { Rapazes (entre } \\
\text { 8-13 anos) com } \\
\text { PHDA (DSM-IV } \\
\text { e confirmação } \\
\text { pelos } \\
\text { Questionários } \\
\text { de Conners para } \\
\text { Pais) } \\
\text { - } n=14 \\
\text { (grupo controlo } \\
n=8 \text { e grupo e } \\
\text { experimental } \\
n=6 \text { ) } \\
\cdot \text { Idade média: } \\
\text { 10,63 anos } \\
\text { - Género (M:F) } \\
=1: 0 \\
\text { - Sob } \\
\text { medicação: } \\
n=14\end{array}$ & $\begin{array}{l}20 \text { Sessões de } \\
\text { ioga (1 hora por } \\
\text { sessão, } 1 \text { vez } \\
\text { semana). } \\
\text { vs } \\
\text { Atividades de } \\
\text { cooperação } \\
\text { (treino de } \\
\text { capacidades de } \\
\text { comunicação, } \\
\text { escuta e partilha) }\end{array}$ & $\begin{array}{l}20 \\
\text { Semanas }\end{array}$ & $\begin{array}{l}\text { Questionários } \\
\text { de Conners para } \\
\text { Pais (CPRS) } \\
\text { Questionários } \\
\text { de Conners para } \\
\text { Professores } \\
\text { (CTRS) }\end{array}$ & $\begin{array}{l}\text { CPRS: } \\
\text { Grupo praticante de ioga - } \\
\text { melhoria significativa em } \\
\text { cinco das subescalas: } \\
\text { labilidade emocional } \\
\text { ( } p=0,001, \text { Cohen's } d 0,79) \text {, } \\
\text { oposição ( } p=0,003, \text { Cohen's } d \\
0,77) \text {, impulsividade }(p=0,008, \\
\text { Cohen's } 0,73) \text {, «índex } \\
\text { global» ( } p=0,019, \text { Cohen's } d \\
0,29) \text { e «índex total» } \\
(p=0,001, \text { Cohen's } d 0,73) \text {. } \\
\text { Grupo controlo- melhoria em } \\
\text { três das subescalas: } \\
\text { hiperatividade ( } p=0,004, \\
\text { Cohen's } d 0,39) \text {, ansiedade } \\
(p=0,028, \text { Cohen's } d 0,59) \text { e } \\
\text { problemas sociais ( }(p=0,034, \\
\text { Cohen's } d 0,85) \text {. } \\
\text { Em ambos, melhoria } \\
\text { significativa nos parâmetros } \\
\text { Perfeccionismo, DSM-IV } \\
\text { Hiperatividade e DSM-IV Total. } \\
\text { CTRS: } \\
\text { Não houve diferença } \\
\text { estatisticamente significativa } \\
\text { para ambos os grupos ( } p>0,05 \\
\text { para todos os parâmetros). }\end{array}$ & . \\
\hline
\end{tabular}

Legenda: $E C A C$ = Ensaio clínico aleatorizado e controlado; PHDA = Perturbação de hiperatividade e défice de atenção; NE = Nível de evidência; DSM = Manual de diagnóstico e estatístico de doenças mentais; CID = Classificação internacional de doenças.

controlo que apresentou melhoria em três parâmetros diferentes: hiperatividade ( $p=0,004$, Cohen's $d 0,39$ ), ansiedade ( $p=0,028$, Cohen's $d 0,59)$ e problemas sociais ( $p=0,034$, Cohen's $d 0,85$ ). Ambos demonstraram melhoria significativa nos parâmetros relativos ao «Perfeccionismo», «DSM-IV Hiperatividade» $\mathrm{e}$ «DSM-IVTotal». No Questionário de Conners para Professores não houve diferença estatisticamente significativa entre os dois grupos - os autores apontam para o facto de a avaliação decorrer durante o período em que as crianças estão sob maior efeito da medicação (tempo escolar), enquanto os pais lidam com as crianças em períodos sem medicação, notando por isso maior diferença a nível de comportamentos. O follow-up limitado do estudo não permite perceber os resultados e os efeitos da prática de ioga mantida ao longo do tempo. As outras limitações metodologicamente encontradas dizem respeito ao facto de ser um estudo sem dupla ocultação, o processo de 
aleatorização não ser descrito e ter uma amostra pequena e heterogénea, particularmente no que diz respeito ao género, idade e grau de escolaridade. Os resultados parecem apontar um papel importante da prática de ioga na estabilização emocional e na redução dos sintomas de oposição, não fornecendo, no entanto, poder estatístico suficiente para se inferirem tais conclusões. Atribuiu-se um nível de evidência 2.

O Quadro II descreve o resumo das principais características dos ensaios clínicos, assim como a avaliação da qualidade e nível de evidência.

\section{CONCLUSÕES}

Na PHDA, a necessidade de encontrar alternativas ou um complemento à terapia farmacológica tem ganho um valor acrescido, uma vez que é uma patologia que exige estratégias de controlo emocional, controlo da impulsividade e treino de atenção, situações que implicam um trabalho individual difícil de gerir apenas com medicação. De uma forma global, os estudos apontam que o ioga atinge o seu efeito benéfico pelo facto de ser uma modalidade que implica disciplina de concentração conjugada com técnicas de atenção e relaxamento. ${ }^{2,6,13}$ Os exercícios praticados no ioga geralmente conduzem a um intenso treino postural, respiratório e mental que pode influenciar positivamente o estado de saúde mental. ${ }^{3,6,13}$ Além disso, está descrito que promovem o autocontrolo, autoestima e redução da tensão corporal, para além de estimularem a atenção e concentração. ${ }^{17}$ Outros estudos referem ainda que a prática de ioga pode ter uma ação positiva na capacidade e/ou função discriminativa das crianças com PHDA por aumentar a atenção e o processamento da informação. ${ }^{3,6}$

No entanto, esta revisão inclui algumas limitações: o tamanho e a heterogeneidade das amostras, nomeadamente no que diz respeito ao género, idade, nível de escolaridade e tipo e dose de medicação. Os próprios programas de ioga também diferiram entre si e, uma vez que é uma modalidade com diversas vertentes, é importante uniformizar o tipo de treino e estudar os exercícios e técnicas mais adequadas para o objetivo em causa. Nas diferentes formas de avaliar os outcomes, a maior parte dos trabalhos apenas tentou estabelecer relação entre a prática de ioga e a diminuição dos sintomas primários de PHDA, não incluindo os sintomas secundários, como as funções cerebrais executivas e dis- criminativas, descritas apenas para o estudo de Chou e Huang $^{6}$ (de 2017). Apontam-se ainda limitações relativas ao período de seguimento de follow-up, diminuindo o poder e a precisão dos estudos, bem como à garantia de aleatorização e dupla ocultação adequadas. A limitação linguística pode eventualmente ter condicionado a não inclusão de estudos que seriam potencialmente relevantes em outras línguas.

Em suma, apesar de terem sido descritos resultados positivos na diminuição dos sintomas de PHDA com a prática de ioga, não existe evidência suficiente para recomendar a sua prática como terapia complementar na PHDA (força de recomendação B) pelas limitações metodológicas apontadas. Não foram encontrados efeitos adversos significativos com a prática de ioga. Em Portugal ainda não foram realizados estudos acerca desta temática. Dada a popularidade da modalidade e a vasta oferta disponível seria pertinente estudar se se adequa ao contexto e realidade portuguesas, sobretudo numa altura em que as terapias alternativas têm sido temas de discussão entre a comunidade médica.

A necessidade da realização, num futuro próximo, de mais estudos aleatorizados, duplamente-cegos, prospetivos e com amostras adequadas torna-se fundamental para perceber o efeito a longo prazo da prática de ioga como terapia complementar em crianças com PHDA. Só assim se poderão obter estudos de boa qualidade que validem a evidência encontrada e auxiliem na formulação de recomendações.

\section{REFERÊNCIAS BIBLIOGRÁFICAS}

1. Saraiva CB, Cerejeira J. Psiquiatria fundamental. Lisboa: LIDEL; 2014. ISBN 9789897520716

2. Lange KM, Makulska-Gertruda E, Hauser J, Reissmann A, Kaunzinger I, Tucha $L$, et al. Yoga and the therapy of children with attention deficit hyperactivity disorder. J Yoga Phys Ther. 2014;4(3):168.

3. Haffner J, Roos J, Goldstein N, Parzer P, Resch F. Zur wirksamkeit körperorientierter therapieverfahren bei der behandlung hyperaktiver Störungen: ergebnisse einer kontrollierten pilotstudie [The effectiveness of body-oriented methods of therapy in the treatment of attention-deficit hyperactivity disorder (ADHD): results of a controlled pilot study]. Z Kinder Jugendpsychiatr Psychother. 2006;34(1):37-47. German

4. American Psychiatric Association. Diagnostic and statistical manual of mental disorders (DSM - 5). 5th ed. American Psychiatric Publishing; 2013. ISBN 9780890425596

5. World Health Organization. The ICD-10 Classification of mental and behavioural disorders: clinical descriptions and diagnostic guidelines. Geneva:WHO; 1992. ISBN 9241544228 
6. Chou CC, Huang CJ. Effects of an 8-week yoga program on sustained attention and discrimination function in children with attention deficit hyperactivity disorder. PeerJ. 2017;5:e2883.

7. Cerrillo-Urbina AJ, García-Hermoso A, Sánchez-López M, Pardo-Guijarro MJ, Santos Gómez JL, Martínez-Vizcaíno V. The effects of physical exercise in children with attention deficit hyperactivity disorder: a systematic review and meta-analysis of randomized control trials. Child Care Health Dev. 2015;41(6):779-88.

8. Harrison LJ, Manocha R, Rubia K. Sahaja yoga meditation as a family treatment programme for children with attention deficit-hyperactivity disorder. Clin Child Psychol Psychiatry. 1998;9(4):479-97.

9. Paris J, Bhat V, Thombs B. Is adult attention-deficit hyperactivity disorder being overdiagnosed? Can J Psychiatry. 2015;60(7):324-8.

10. Taylor E. Attention deficit hyperactivity disorder: overdiagnosed or diagnoses missed? Arch Dis Child. 2017;102(4):376-379.

11. Galantino ML, Galbavy R, Quinn L. Therapeutic effects of yoga for children: a systematic review of the literature. Pediatr Phys Ther. 2008;20(1): 66-80.

12. Balasubramaniam M, Telles S, Doraiswamy PM. Yoga on our minds: a systematic review of yoga for neuropsychiatric disorders. Front Psychiatry. 2013;3:117.

13. Abadi MS, Madgaonkar J, Venkantesan S. Effect of yoga on children with attention deficit/hyperactivity disorder. Psychol Stud. 2008;53(2):154-9.
14. Birdee GS, Yeh GY, Wayne PM, Phillips RS, Davis RB, Gardiner P. Clinical applications of yoga for the pediatric population: a systematic review. Acad Pediatr. 2009;9(4):212-20.e1-9.

15. Ebell MH, Siwek J, Weiss BD, Woolf SH, Susman J, Ewigman B, et al. Strength of recommendation taxonomy (SORT): a patient-centered approach to grading evidence in the medical literature. Am Fam Physician. 2004;69(3):548-56.

16. Jensen PS, Kenny DT. The effects of yoga on the attention and behavior of boys with attention-deficit/hyperactivity disorder (ADHD). J Atten Disord. 2004;7(4):205-16.

17. Peck HL, Kehle T, Bray MA, Theodore LA. Yoga as an intervention for children with attention. School Psych Rev. 2005;34(3):415-24.

\section{CONFLITO DE INTERESSES}

Os autores declaram não ter quaisquer conflitos de interesse.

\section{ENDEREÇO PARA CORRESPONDÊNCIA}

Inês Pintalhão

E-mail: inesmspintalhao@gmail.com

http://orcid.org/0000-0002-6143-9960

Recebido em 21-04-2017

Aceite para publicação em 05-01-2019

\section{ABSTRACT \\ YOGA PRACTICE AS A COMPLEMENTARY OR ALTERNATIVE THERAPY IN CHILDREN WITH ATTENTION DEFICIT HYPERACTIVITY DISORDER: AN EVIDENCE-BASED REVIEW}

Objectives: To review the evidence available on the effectiveness of yoga practice in the reduction of ADHD symptoms in children and adolescents.

Data source: MEDLINE/PubMed and evidence-based medicine databases (National Guideline Clearinghouse, Canadian Medical Association Practice Guidelines InfoBase, Guidelines Finder of the National NHS British Library for Health, DARE, Bandolier, and The Cochrane Library).

Methods: We conducted an evidence-based review of scientific papers published between January 1999 and January 2017, in Portuguese and English, using the MeSH terms 'attention deficit hyperactivity disorder' (ADHD), 'yoga', 'child', and 'adolescent'. Levels of evidence (LE) and strength of recommendations (SOR) were assigned according to the American Family Physician's Strength of Recommendation Taxonomy (SORT) criteria.

Results: Our search revealed a total of 48 papers and we selected five according to the inclusion criteria: one systematic review and four original articles, of which three randomised controlled trials, and one quasi-experimental trial. Although some studies have shown positive results, there is insufficient evidence to demonstrate a beneficial effect of yoga practice on the reduction of symptoms in children and adolescents with ADHD (LE 2).

Conclusion: The evidence available does not support the recommendation of yoga practice as an alternative or complementary therapy to the strategies already implemented, in children and adolescents with ADHD (strength of recommendation B). Studies that point out to a beneficial effect of this modality raise concerns about their methodology, limiting the quality of the evidence. Further prospective large-scale studies, with homogeneous samples and adequate follow-up are suggested, in order to allow the validation of the evidence found to date, and to help in the formulation of future recommendations.

Keywords: Attention deficit hyperactivity disorder; Yoga; Child; Adolescent. 